\title{
AVALIAÇÃO DOS RESULTADOS PÓS-CIFOSECTOMIA NA MIELOMENINGOCELE PELA TÉCNICA DE DUNN-MCCARTHY MODIFICADA
}

\author{
EVALUATION OF RESULTS AFTER KYPHECTOMY IN MYELOMENINGOCELE \\ BY THE MODIFIED DUNN-MCCARTHY TECHNIQUE
}

\section{EVALUACIÓN DE LA POST-CIFOSECTOMÍA EN MIELOMENINGOCELE POR TÉCNICA DE DUNN-MCCARTHY MODIFICADA}

Rafael Cantarelli dos Santos', Paulo Tadeu Maia Cavali², Maurício Antonelli Lehoczki ${ }^{3}$, Alexander Junoueira Rossato ${ }^{3}$, Marcelo Italo Risso-Neto ${ }^{4}$, Guilherme Rebechi Zuiani ${ }^{4}$, Ivan Guidolin Veiga ${ }^{4}$, Wagner PasoualinI ${ }^{4}$, Élcio Landim ${ }^{5}$

\section{RESUMO}

Objetivo: Avaliar os resultados do tratamento cirúrgico da cifose lombar congênita em portadores de mielomeningocele. Métodos: Realizado estudo retrospectivo incluindo 31 pacientes, com média de idade de 10 anos, tratados cirurgicamente por via posterior exclusiva pela técnica de Dunn-McCarthy. O segmento médio foi de 22 meses (5 a 60). Foram avaliados os potenciais de correção, perda de correção, percentuais de fusão e complicações. Resultados: A cifose inicial média de $114^{\circ}\left(60^{\circ}\right.$ a $\left.183^{\circ}\right)$ obteve correção média para $37^{\circ}$ $\left(-63^{\circ}\right.$ a $\left.180^{\circ}\right)$ na radiografia obtida na última consulta. A taxa global de complicações foi de $46 \%$ entretanto a morbidade cirúrgica foi muito baixa. A taxa de fusão de $80 \%$. Conclusão: A técnica descrita se mostrou eficiente, com um alto potencial de correção e fusão, e com baixo potencial de perda de correção.

Descritores: Meningomielocele; Cifose; Resultado de tratamento; Estudos retrosprospectivos.

\section{ABSTRACT}

Objective: To report the results of the surgical treatment of congenital lumbar kyphosis related to myelomeningocele. Methods: Retrospective study, with 31 patients, mean age of 10 years old, treated only by posterior approach with a Dunn-McCarthy procedure. The mean follow up was about 22 months (5 to 60 months). We evaluated the potential correction, correction loss, percentage of fusion and complications. Results: The average preoperative kyphosis of $114^{\circ}\left(60^{\circ}\right.$ to $\left.183^{\circ}\right)$ was corrected to $37^{\circ}\left(-63^{\circ}\right.$ to $\left.180^{\circ}\right)$ according to the radiograph obtained in the last visit. The global rate of complications was 46\%; however the surgical morbidity was very low. The fusion rate was $80 \%$. Conclusion: This procedure is efficient, with a high potential for correction and fusion, and low potential for loss of correction.

Keywords: Meningomyelocele; Kyphosis; Treatment outcome; Retrospective studies.

\section{RESUMEN}

Objetivo: Evaluar los resultados del tratamiento quirúrgico de la cifosis congénita en pacientes con mielomeningocele lumbar. Métodos: Estudio retrospectivo de 31 pacientes con una edad media de 10 años, tratados quirúrgicamente por acceso exclusivamente posterior por la técnica de Dunn-McCarthy modificada. El seguimiento medio fue de 22 meses (50-60). Se evaluó el potencial de corrección, la pérdida de la corrección, el porcentaje de fusión y las complicaciones. Resultados: La cifosis inicial promedio de $114^{\circ}\left(60^{\circ}\right.$ a $\left.183^{\circ}\right)$ obtuvo corrección promedio a $37^{\circ}\left(-63^{\circ}\right.$ a $\left.180^{\circ}\right)$ en las radiografías obtenidas en la última visita. La tasa global de complicaciones fue del $46 \%$, sin embargo, la morbilidad quirúrgica fue muy baja. La tasa de fusión fue el $80 \%$. Conclusión: La técnica descrita ha demostrado su eficacia, con un alto potencial para la corrección y fusión, y bajo potencial de pérdida de corrección.

Descriptores: Meningomielocele; Cifosis; Resultado del Tratamiento; Estudios Retrospectivo.

\section{INTRODUÇÃO}

A incidência da cifose congênita nos pacientes portadores de mielomeningocele é de 8 a 15\% $\%^{1,2}$. Esta deformidade, proveniente de uma anomalia do crescimento vertebral, leva a uma deformidade progressiva e incapacitante com inúmeras repercussões clínicas cujo tratamento é um desafio para os cirurgiões devido ao grande número de complicações.
A deformidade progressiva e em ângulo agudo muitas vezes leva a formação de escaras de difícil manejo pela dificuldade de cicatrização e infecções recorrentes. Associado a isto, ainda observamos uma dificuldade do sentar em cadeiras devido a um desequilíbrio do balanço sagital provocando dificuldade no livre movimento dos membros superiores, já que os mesmos são utilizados como apoio para se manter em uma posição adequada.

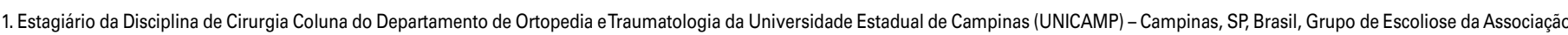
de Assistência à Criança Deficiente - AACD - São Paulo, SP, Brasil.

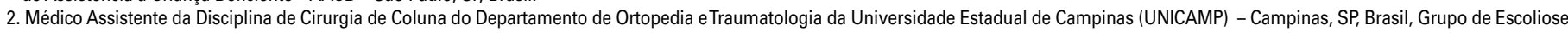
da Associação de Assistência à Criança Deficiente - AACD - São Paulo, SP, Brasil.

3. Médico Assistente do Grupo de Escoliose da Associação de Assistência à Criança Deficiente (AACD) - São Paulo, SP, Brasil.

4. Médico Assistente da Disciplina de Cirurgia de Coluna do Departamento de Ortopedia eTraumatologia da Universidade Estadual de Campinas (UNICAMP) - Campinas, SP, Brasil.

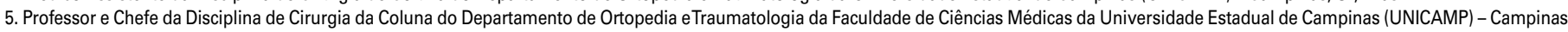
SP, Brasil, Chefe do Grupo de Escoliose da Associação de Assistência à Criança Deficiente (AACD) - São Paulo, SP, Brasil. 
Tais fatores causam uma piora do quadro psicológico da criança, pois impedem uma maior integração social ${ }^{1,3}$

Outros problemas que ainda podem ser citados são as alterações respiratórias e urinárias geradas por uma diminuição do volume abdominal causada por uma hiperpressão proveniente da flexão exagerada do tronco a qual pode comprimir o diafragma e os ureteres ${ }^{4}$.

Seu tratamento é complexo. Os métodos conservadores, como uso de braces, são restritos, pois não são capazes de reduzir ou minimizar a progressão da curva, além de serem pouco tolerados e levarem a um aumento da formação de escaras. Portanto, o tratamento cirúrgico é o método mais eficaz no controle e correção da deformidade ${ }^{5,6}$. Porém, ainda um desafio devido à má qualidade cutânea do dorso, deformidades rígidas, ossos osteopênicos e índices elevados de pseudoartrose e infecção proveniente muitas vezes da colonização do trato urinário presentes na maioria dos doentes.

O objetivo deste trabalho é avaliar a correção cirúrgica da cifose congênita em pacientes portadores de mielomeningocele, utilizando-se exclusivamente a via posterior e realizando a ressecção de uma ou mais vértebras do ápice da deformidade associada a instrumentação rígida.

\section{MATERIAIS E MÉTODOS}

Do período de março de 2002 a junho de 2010, quarenta e três pacientes portadores de cifose congênita rígida associada a mielomeningocele foram submetidas ao tratamento cirúrgico pela técnica de Dunn-McCarthy modificada no Hospital Abreu Sodré, sede da Associação de Assistência a Criança Deficiente (AACD) em São Paulo. Realizada revisão retrospectiva com avaliação do prontuário hospitalar, evolução clínica, exame físico descrição cirúrgica, radiografias e fotografias.

Dentre os 43 pacientes selecionados 12 foram excluídos devidos a falta de dados completos restando uma amostra de 31 pacientes no total. Destes, 15 eram do sexo masculino e 16 do sexo feminino, com média de idade de 10 anos no dia do ato cirúrgico. Nenhum paciente apresentava função motora abaixo do nível torácico sendo o grupo formado exclusivamente por não deambuladores. Todos receberam tratamento neurocirúrgico com fechamento da bolsa da mielomeningocele imediatamente após o nascimento. Todos os pacientes com indicação cirúrgica foram submetidos a uma criteriosa investigação pré-operatória que incluiu teste de alergia ao látex, por meio do antígeno do látex k82 usado para detectar IgE específicos pelo método enzimaimunofluorimétrico, exame de urina tipo I e urocultura. Do sangue, foram analisados uréia, creatinina, sódio, potássio, glicemia de jejum, hemograma completo e coagulograma. Todos fizeram radiografia de tórax póstero-anterior. Após a realização dos exames foram submetidos a uma avaliação pré-operatória pelo pediatra.

Por critério da instituição, apenas os pacientes com peso superior a $20 \mathrm{~kg}$ e bom estado nutricional foram submetidos a esse tratamento em virtude do grande volume de sangramento deste tipo de procedimento cirúrgico.

Por motivo de preservação parcial do desenvolvimento pulmonar, a idade mínima preconizada para o procedimento foi de cinco anos

Foram avaliadas as radiografias do perfil e antero-posterior obtidas sempre na posição sentada e numa segunda sessão com tração no pré-operatório.Em todas a incidências foi realizada a medida da cifose pelo ângulo de COBB, de modo a quantificar em graus positivos a cifose e negativo a lordose.

\section{TÉCNICA CIRÚRGICA}

A via de acesso utilizada foi sempre a posterior no sentido longitudinal da região torácica até S2 com pequenos desvios na região de fechamento da mielomeningocele evitando-se as regiões de pele menos vascularizadas. Iniciando-se em uma região onde a anatomia era preservada seguiu-se em direção ao ápice da deformidade até o saco dural com cautela para evitar lesões e fístulas liquóricas.
Em seguida foi isolado e ligado o saco dural em suas duas extremidades expondo todo o canal vertebral desde a vértebra apical até S2 (Figura 1). A discectomia é realizada nos níveis adjacentes à vertebrectomia.

A vertebrectomia consiste da ressecção de uma a quatro vértebras no ápice da deformidade com preservação do ligamento longitudinal anterior, sendo que, quanto maior a rigidez e o grau de cifose, maior o número de vértebras a ser ressecadas.

A instrumentação com duas hastes de $5 \mathrm{~mm}$ moldadas em "S" distal - DUNN (Figura 2) tem por finalidade obter uma alavanca. Para isso, introduzimos a porção distal da haste no forâmen de S1 e seguimos com a fixação das vértebras, de distal para proximal, reduzindo a deformidade cifótica com a manobra de "Cantilever". Neste passo, a moldagem é realizada antes do inicio da cirurgia com base em uma radiografia de perfil, ganhando tempo cirúrgico e evitando um maior sangramento. Efetuamos a instrumentação com amarriasublaminar associada a duas hastes de aço pela técnica de Luque ou com parafusos pediculares e hastes de titânio.

A amarriasublaminar foi realizada de maneira clássica na região proximal à espinha bífida onde as vértebras possuem lâminas normais e quando possível também distal.Os parafusos torácicos foram introduzidos desde a coluna normal até as vértebras que apresentavam espinha bífida.

A decorticação foi realizada de maneira criteriosa envolvendo a lâmina e apófises articulares remanescentes na vértebra com espinha bífida promovendo assim, um leito ósseo com maior potencial de consolidação para o enxerto ósseo autólogo. A colocação de enxerto também foi feita nos espaços onde os discos foram retirados, realizando uma artrodese intersomática desde o ápice da vertebrectomia até o espaço intervertebral de L5-S1.

Para se obter uma maior estabilidade dois ou um dispositivo de travamento transversais foram colocados nas extremidades da montagem, evitando a rotação das hastes principalmente na região distal onde podem gerar lesões de estruturas intrapélvicas.

Os parâmetros avaliados neste trabalho foram os índices de complicações, os potenciais de correção, potenciais de perda de correção, percentual de fusão, grau de satisfação da família e do paciente

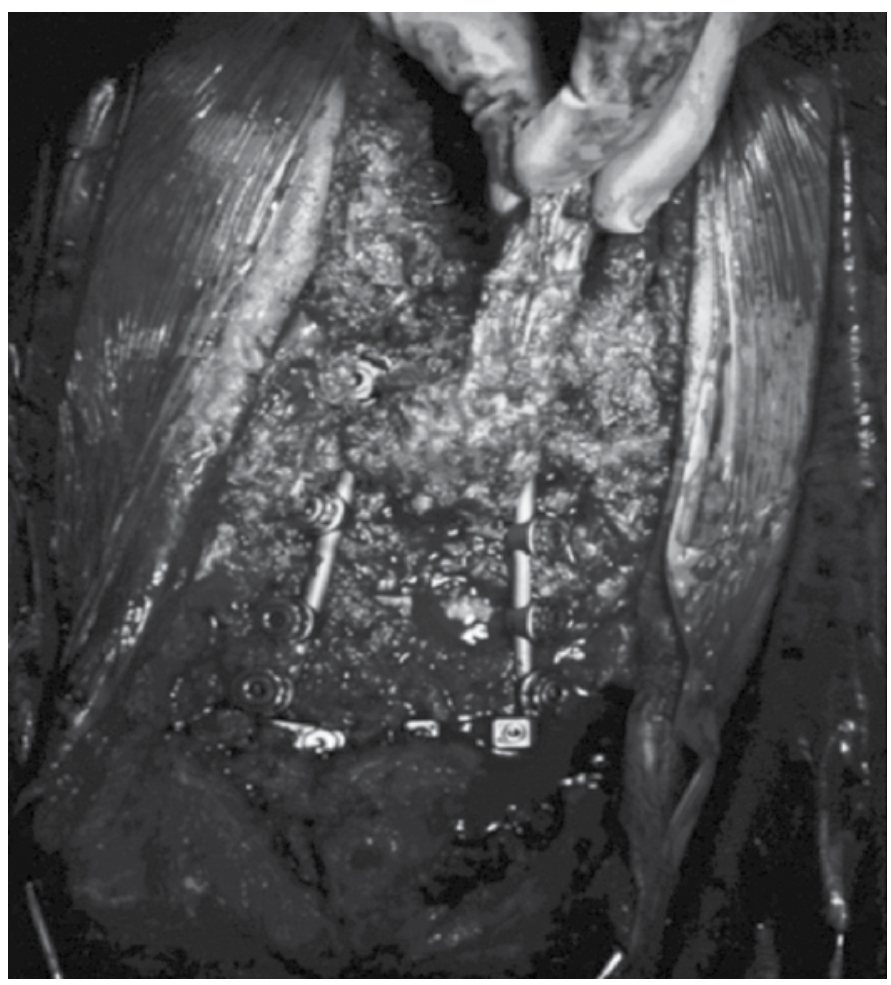

Figura 1. Saco Dural rebatido superiormente.

Fonte: AACD. 


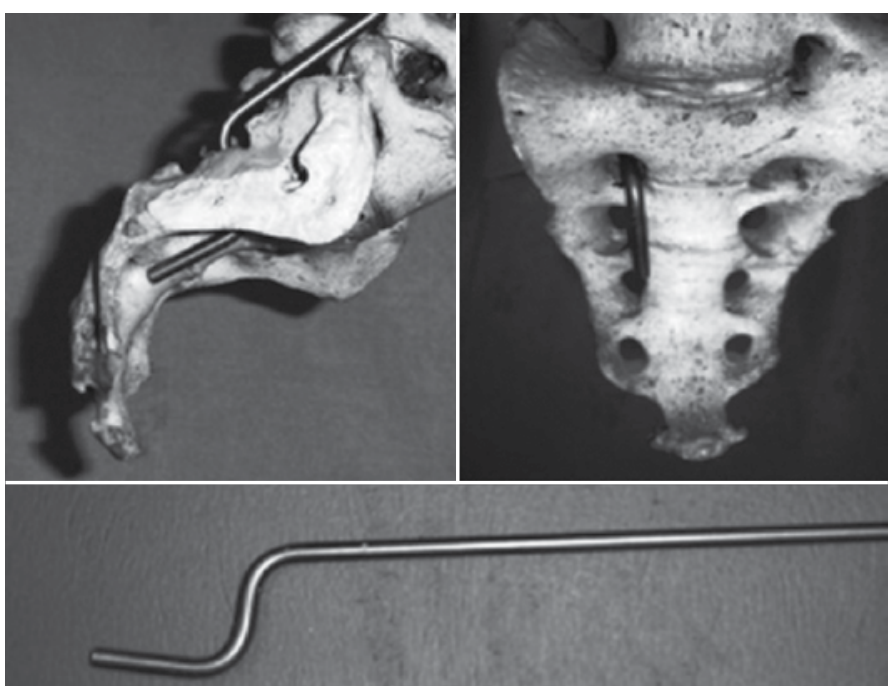

Figura 2. Haste moldada em "S" distal introduzida no primeiro forame do sacro, nas posições perfil e antero-posterior, respectivamente.

Fonte: AACD.

além da melhora clínica obtida. Tais parâmetros foram avaliados com base na medida dos ângulos de cifose e exame físico onde avaliamos a capacidade funcional do paciente em relação ao livre movimento dos membros superiores e capacidade de sentar adequadamente.

\section{RESULTADOS}

Dos 31 pacientes estudados 15 eram do sexo masculino e 16 do feminino, a idade variou de 6 a 17 anos sendo a média de 10 anos e o tempo médio de seguimento foi de 22 meses com variações de 5 a 60 .

A cifose média no pré-operatório era de $114^{\circ}\left(60^{\circ}\right.$ a $\left.183^{\circ}\right)$ e foi corrigida para $37^{\circ}\left(-63^{\circ}\right.$ a $\left.180^{\circ}\right)$ (Figuras 3 e 4). A correção média foi de $90^{\circ}$ e a perda de correção $10^{\circ}$ comparando com o pós operatório inicial e a última visita ambulatorial. O ápice da cifose foi T12 em um paciente, L1 em 11, L2 em 9 e L3 em 10.

A taxa global de complicações foi de $46 \%$, com cinco casos de infecção, sendo 2 superficiais, 2 profundas e 1 caso de meningite; 3 casos de necroses de pele; 1 de hematoma; 1 procedimento cirúrgico foi interrompido devido a sangramento excessivo; 5 pseudoartroses sendo $4 \mathrm{com}$ soltura e quebra do material e 1 por infecção seguida de meningite que necessitou da retirada do material de síntese.

Nos pacientes N3, N10 e N14, em que foi evidenciada pseudoartrose com quebra ou soltura do material, foi optado pela retirada do material de síntese sem nova reinstrumentação e apenas seguimento ambulatorial. O paciente N12 que teve como complicação a pseudoartrose com soltura do Dunn foi reoperado com troca da instrumentação para parafusos pediculares com boa evolução. 0 N28 que apresentou infecção profunda e meningite foi tratada com desbridamento cirúrgico, retirada do implante e antibiótico-terapia com normalização das provas inflamatória e no momento aguarda nova programação cirúrgica.

O paciente N16 apresentou infecção profunda que culminou na retirada do implante após 1 ano e nova intervenção cirúrgica com rotação de retalho músculo-cutâneo pelo cirurgião plástico, curativos e antibiótico-terapia. As infecções superficiais nos pacientes N4 e N5 e necroses de pele dos N1 e 19 foram tratadas com êxito em um curto espaço de tempo por meio de curativos diários. Nas lesões mais profundas, os curativos foram realizados com fita de alginato de cálcio e sessões de câmara hiperbárica e nas mais superficiais com pouco secreção foram utilizadas placas de hidrocolóide sendo as trocas realizadas a cada 72 horas. 0 desbridamento das lesões foi efetuado com soro fisiológico a 0,9\% levemente aquecido lançado sob pressão com seringa de $60 \mathrm{ml}$ e

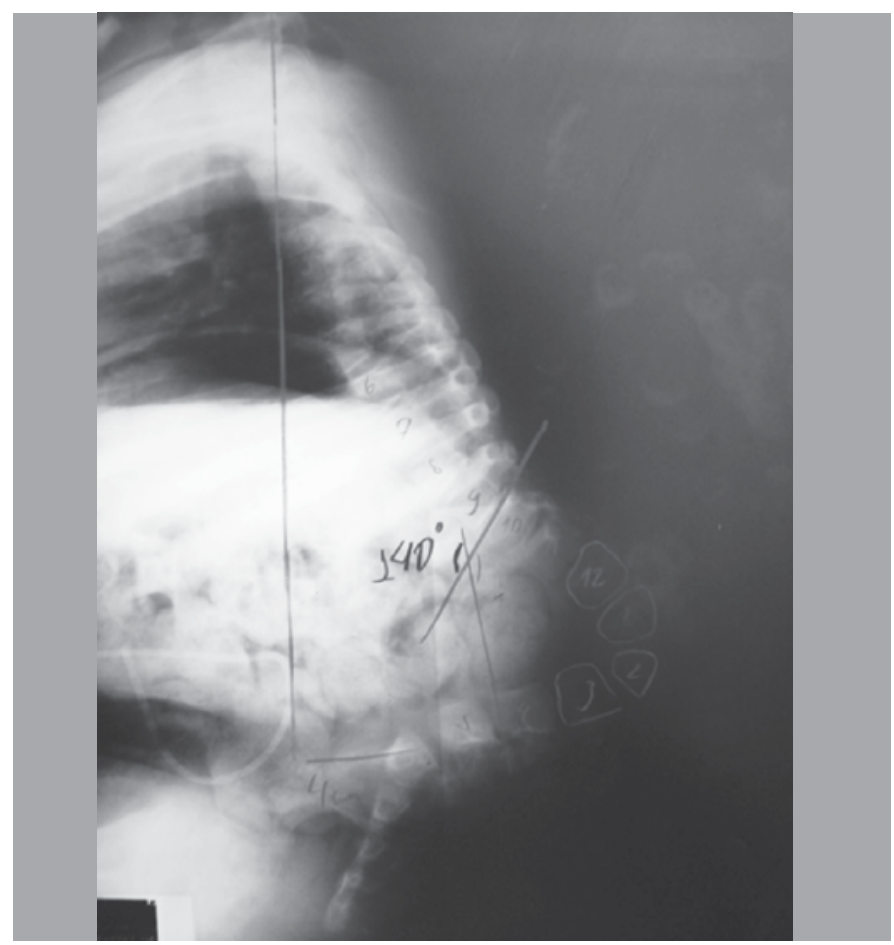

Figura 3. Cifose no pré operatório.

Fonte: AACD.

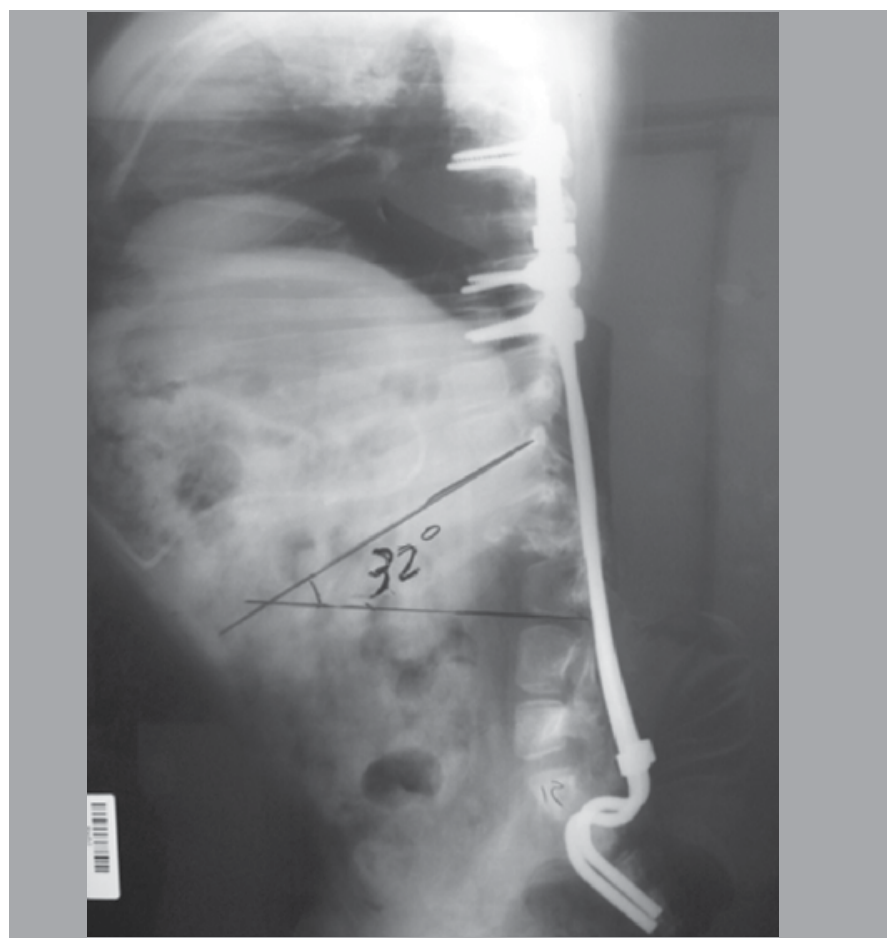

Figura 4. Resultado pós-operatório.

Fonte: AACD

agulha 40X12. O hematoma ocorrido no paciente N9 foi drenado precocemente com boa evolução.

A taxa de fusão foi de $80 \%$, a grande maioria dos pacientes e familiares ficaram muito satisfeitos com os resultados obtidos como melhora da deformidade, ulcerações cutâneas e principalmente pela capacidade plena de sentar de forma independente e manipular objetos com as mãos sem a necessidade de utilizar os membros superiores como apoio. 


\section{DISCUSSÃO}

O tratamento cirúrgico da cifose congênita na mielomeningocele ainda é um desafio para a maioria dos cirurgiões por se tratar de uma deformidade rígida de difícil correção associada a muitas complicações com altas taxas de infecção, pseudoartrose, necrose cutânea. Isso se deve a má qualidade e vascularização da pele do dorso, dificuldade na instrumentação devido ao osso osteopênico, grandes perdas sanguíneas, infecções urinárias de repetição e alergia ao látex.

Os primeiros relatos do tratamento cirúrgico desta deformidade foram realizados por Sharrard e Drennan ${ }^{7}$, que descreveu pela primeira vez a osteotomia e artrodese para correção da cifose em neonatos com mielomeningocele. A partir de então outros autores passaram a publicar seus casos com técnicas variadas com via anterior, posterior e combinada, porém todas elas esbarravam na mesma dificuldade que seria de atingir e manter uma boa correção da deformidade com um mínimo de complicações e morbidade.

A técnica de Dunn-McCarthy et al. ${ }^{8}$ modificada, descrita neste trabalho, se mostrou eficaz em atingir uma boa correção cirúrgica com altas taxas de fusão e pequenas perdas de correção. Além de utilizar apenas a via de acesso posterior reduzindo o tempo cirúrgico e volume de sangramento e infecção.

Em 1994, Litner e Lindseth² publicaram um estudo com 39 pacientes relatando o tratamento cirúrgico desta deformidade com uma cifose pré-operatória média de 111 que foi corrigida para 40 e mantida em 62. McCall ${ }^{3}$ em 1998, com 16 pacientes evidenciaram uma cifose média 111 corrigida para 15 e mantida em 20. Hung e Lubicky ${ }^{9}$ uma cifose de 126 corrigida para 21 e mantida em 23. De forma semelhante, em nossa amostra de 31 pacientes observamos uma cifose inicial média de 114 corrigida para 28 e mantida em 37.

Nosso índice de infecção e lesões de pele foi de $26 \%$ ficando abaixo dos índices observados na literatura. Na série de Hull et al. ${ }^{10}$ tais complicações chegaram a $45 \%$.

Autores relataram três casos de anafilaxia ao látex em um trabalho relacionado à mielomeningocele ${ }^{11}$. No Hospital Abreu Sodré, sede da AACD, um trabalho com 187 pacientes portadores de mielomeningocele mostrou um índice de 29,5\% de alérgicos ao látex ${ }^{12}$. Em nossa casuística, não obtivemos nenhum caso de anafilaxia, pois todos os pacientes foram submetidos ao teste, que, se positivo, determinava o preparo do centro cirúrgico em um ambiente $100 \%$ "látex free".

Ao contrário da maioria dos outros trabalhos, nossa idade média para cirurgia foi um pouco superior ao da literatura por critérios da instituição que determinava: uma idade mínima de 5 anos, para permitir melhor desenvolvimento pulmonar; e peso mínimo de $20 \mathrm{~kg}$ e bom estado nutricional, devido aos grandes volumes de sangramento que atingiam até mesmo a troca da volemia de criança.

Outro fato a ser levado em conta que diverge da literatura é a realização da cordectomia realizada em todos nossos pacientes sem evidência de aumento de infecção e alteração do fluxo liquórico $^{3}$. Obtivemos apenas um caso de meningite.

\section{CONCLUSÃO}

A técnica de Dunn-McCarthy modificada é uma opção segura e eficaz no tratamento da cifose congênita associada a mielomeningocele, demonstrada pelo alto percentual de fusão e correção deformidade com pouca perda de correção durante o seguimento. Alcançou os objetivos em propiciar ao paciente uma melhora do sentar sem necessidade do apoio dos membros superiores além da resolução das ulcerações cutâneas do dorso atingindo alto grau de satisfação dos pacientes e familiares.

\section{REFERÊNCIAS}

1. Lindseth RE, Stelzer L Jr. Vertebral excision for kyphosis in children with myelomeningocele. J Bone Joint Surg Am. 1979;61(5):699-704.

2. Lintner SA, Lindseth RE. Kyphotic deformity in patients who have a myelomeningocele. Operative treatment and long-term follow-up. J Bone Joint Surg Am. 1994;76(9):1301-7.

3. McCall RE. Modified luque instrumentation after myelomeningocelekyphectomy. Spine (PhilaPa 1976).1998;23(12):1406-11.

4. McMaster MJ. The long-term results of kyphectomy and spinal stabilization in children with myelomeningocele. Spine (Phila Pa 1976). 1988;13(4):417-24.

5. Banta JV, Hamada JS. Natural history of the kyphotic deformity in myelomeningocele. J Bone Joint Surg Am. 1976;58:279.

6. Torode I, Godette G. Surgical Correction of congenital kyphosis in myelomeningocele. J PediatrOrthop. 1995;15(2):202-5.
7. Sharrard WJW, Drennan JC. Osteotomy: excision of the spine for lumbar kyphosis in older children with myelomeningocele. J Bone Joint Surg Br.1972;54:50-60.

8. McCarthy RE, Bruffett WL, McCullough FL. S rod fixation to the sacrum in patients with neuromuscular spinal deformities. ClinOrthopRelat Res. 1999; (364):26-31.

9. Huang TJ, Lubicky JP. Kyphectomy and segmental spinal instrumentation in young children with myelomeningocele kyphosis. J Formos Med Assoc. 1994; 93(6):503-8.

10. Hull WJ, Moe JH, Lai C, Winter RB. The surgical treatment of spinal deformities in myelomeningocele. J Bone Joint Surg Am. 1975;57:1767.

11. Pierz K, Banta J, Thomson J, Gahm N, Hartford J. The effect of tethered cord release on scoliosis in myelomeningocele. J PediatrOrthop. 2000;20(3):362-5

12. Fernandes $A C$, Bitu SOB, Violante Júnior H. Alergia ao látex em pacientes portadores de mielomeningocele. Rev Bras Ortop. 2006;41(6):217-20. 